\title{
Ciudadanos y gobierno electrónico: la orientación al ciudadano de los sitios Web municipales en Colombia para la promoción de la participación ${ }^{1}$
}

\author{
Sebastián Lippez-De Castro \\ Pontificia Universidad Javeriana, Bogotá, Colombia ${ }^{3}$ \\ slippez@javeriana.edu.co \\ Roberto Garcia Alonso4 \\ Pontificia Universidad Javeriana, Bogotá, Colombia ${ }^{5}$ \\ garcia.roberto@javeriana.edu.co \\ Recibido: 16 de julio de 2015 \\ Aceptado: 21 de octubre de 2015 \\ Disponible en línea: 9 de mayo de 2016
}

Artículo de investigación registrado en el plan de trabajo de los autores en su condición de profesores de planta del Departamento de Ciencia Politica de la Pontificia Universidad Javeriana.

2 Master en Administración Pública con certificado en Gerencia de Gobiernos Locales de la Universidad del Estado de Nueva York en Binghamton University. Politólogo con énfasis en Gestión Pública y Especialista en Gobierno Municipal de la Pontificia Universidad Javeriana.

3 Profesor de planta del Departamento de Ciencia Política de la Pontificia Universidad Javeriana. Director de la Carrera de Ciencia Política.

4 Doctor en Ciencias Políticas y de la Administración, M.A en Democracia y Gobierno de la Universidad Autónoma de Madrid. Politólogo y licenciado en Derecho de la Autónoma de Madrid. Ha impartido docencia en la Universidad del Rosario (Bogotá-Colombia) y ha sido visiting scholar en las Universidades de Brown. Es miembro del Centro de Teoría Política de la UAM (CTP) y miembro del Grupo de Investigación de Teoría Política de la UAM y de la Línea de Investigación en Participación política ciudadana de la Facultad de Ciencia Política y Relaciones Internacionales.

5 Profesor Asistente en la Facultad de Ciencia Politica y Relaciones Internacionales de la Pontificia Universidad Javeriana. 


\title{
Ciudadanos y gobierno electrónico: la orientación al ciudadano de los sitios Web municipales en Colombia para la promoción de la participación
}

\begin{abstract}
Resumen
La adopción, uso y difusión de las Nuevas Tecnologias de la Información y la Comunicación (NTICs) dentro de las organizaciones públicas ha sido una cuestión del máximo interés durante los últimos años, entre otras cosas porque de ello se espera una determinada modificación en las relaciones Estado-sociedad. En contraste con las perspectivas que sobrevaloran el papel de la tecnología en la promoción de una administración pública más abierta y eficaz, el artículo explora una dimensión importante para un pleno desarrollo de las potencialidades que se otorgan a estas nuevas tecnologías, la integración del aspecto humano. El análisis entonces, se concentra en la potencialidad del gobierno electrónico para fomentar la participación ciudadana. Para ello, se revisaron los sitios web de los 32 municipios capitales de departamento en Colombia y se evaluó la presencia de una batería de características orientadas a la provisión de información, la consulta ciudadana, y la participación y compromiso activo ciudadano.

Palabras clave: gobierno electrónico; participación activa; Nuevas Tecnologias de la Información y la Comunicación; e-deliberación; compromiso ciudadano; Gobierno en Línea
\end{abstract}

\section{Citizens and Electronic Government: Colombian Municipal Website Citizen Guidance to Promote Participation}

\begin{abstract}
The adoption, use, and spread of the New Information and Communication Technologies (NTICs) in public organizations has been a top interest matter during the last few years; among others, because from them it is expected a certain change in the relationship between State and society. In contrast with perspectives that overvalue the role of technology in promoting a more open and efficient public management, this article explores an important dimension for a full development of potentialities granted to these new technologies: the integration of the human aspect. So, the analysis focuses on the potentiality of the electronic government to foster citizen participation. For this purposes we reviewed the websites of the 32 capital municipalities of the departments of Colombia and we assessed the presence of a cluster of characteristics aimed to provide information, citizen consultation, and an active citizen participation and commitment. Keywords: electronic government; active participation; new information and communication technologies; e-deliberation; citizen commitment; government online
\end{abstract}

\section{Cidadãos e governo eletrônico: a orientação aos cidadãos dos sites municipais na Colômbia para a promoção da participação}

\section{Resumo}

A adopção, uso e difusão das Novas Tecnologias da Informação e Comunicação (NTICs) dentro das organizações públicas já foi um assunto do máximo interesse durante os últimos anos, em parte por causa de uma esperada certa mudança nas relações Estado-sociedade. Em contraste com as perspectivas que superestimam o papel da tecnologia na promoção de uma administração pública mais aberta e eficaz, o artigo explora uma dimensão importante para o pleno desenvolvimento das potencialidades outorgadas a essas novas tecnologias, a integração do aspecto humano. A análise então, centra na potencialidade do governo eletrônico para fomentar a participação cidadã. Para isso, revisitaram-se os sites dos 32 municipios capitais de departamento na Colômbia e avaliou-se a presença de um grupo de características orientadas ao fornecimento de informação, consulta cidadã e participação e engajamento ativo cidadão. Palavras-chave: governo eletrônico; participação ativa; Novas Tecnologias da Informação e Comunicação; e-deliberação; engajamento cidadão; Governo on-line 
Aunque de acuerdo con la más reciente Encuesta de las Naciones Unidas sobre Gobierno Electrónico (United Nations, 2014), Uruguay y Chile ostentan los puestos de liderazgo en Sur América, Colombia sigue ocupando un lugar destacado en la región. Esta encuesta ubica al país en el puesto 50 del Ranking de 2014 con un 'alto' índice de desarrollo del gobierno electrónico por encima del promedio mundial y regional. Por su parte, la Comisión de Regulación de Comunicaciones (2014) destaca en su segundo informe de Indicadores Sectoriales de las Tecnologías de la Información y las Comunicaciones, "Avance de Colombia en la Sociedad de la Información”, que el 100\% de las organizaciones públicas tienen presencia en la red y ofrecen servicios en línea. En el mismo sentido, la mencionada Encuesta de las Naciones Unidas sobre Gobierno Electrónico (2014) resalta la existencia del Centro para la Innovación sobre el Desarrollo del Gobierno Electrónico en Colombia y el portal de transparencia del gobierno nacional denominado Urna de Cristal, como experiencias destacadas en la región. Una y otra demuestran la intención del gobierno colombiano de adelantar iniciativas de promoción del gobierno electrónico tanto a nivel nacional como subnacional.

Este creciente interés por promover estrategias de gobierno electrónico en Colombia se circunscribe a una tendencia global y a la expectativa general sobre las potencialidades derivadas de la adopción de las Nuevas Tecnologías de la Información y la Comunicación (NTICs) en los procesos gubernamentales. Parece haberse extendido la impresión generalizada de que estas herramientas, las NTICs y las innovaciones tecnológicas en general, suponen una transformación sustantiva de las relaciones Estado-sociedad en la medida en que permitiren resolver muchos de sus problemas e incluso facilitar nuevas formas de gobierno más democrático y participativo. Helbig, Gil-García y Ferro (2009) identifican que los defensores del gobierno electrónico "con frecuencia prometen el impacto de un mejor gobierno, incluyendo un incremento en la calidad de los servicios, ahorros en costos, una más amplia participación política, o políticas y programas más efectivos" (p. 89). En el mismo sentido, Holzer, Melitski, Rho, y Schwester (2004) sostienen que "las TIC's tienen el potencial de hacer que la participación ciudadana sea un elemento más dinámico del proceso 
de política pública" (p. 4). Además, no es raro encontrar aparejadas la implementación de NTICs con la idea de 'buen gobierno', ya que la provisión de información sobre el Estado y los asuntos públicos a través de estos canales, en últimas ayudaría a la transparencia, eficiencia, legitimidad y gobernabilidad.

Se asume entonces que el papel de internet y de las nuevas tecnologías plantean numerosos beneficios y que estos en muchos casos van más allá de la provisión de servicios en línea y la consulta a los ciudadanos sobre determinadas preferencias, se prevé que podría incrementar la participación y deliberación ciudadana sobre los asuntos públicos y con ello la posibilidad de un gobierno más eficaz, eficiente, transparente y participativo. De alli que se plantee incluso una reconfiguración de las relaciones Estado-sociedad mucho más orientadas a los ciudadanos, con mayores dificultades para la centralización y el monopolio de la información y las decisiones en manos de pocas autoridades, y con la posibilidad de revitalizar las pretensiones de una mayor participación politica directa. Pero por supuesto esta perspectiva ha encontrado quienes llamen la atención sobre las dificultades de alcanzar los beneficios esperados, bien por las condiciones estructurales que condicionan el acceso, la accesibilidad y el uso apropiado de las NTICs -es decir de la denominada brecha digital (ver por ejemplo Hacker, Mason y Morgan, 2009; Helbig, Gil-García y Ferro, 2009; Rubaii-Barrett y Wise, 2006; 2008) - o bien por otros argumentos asociados al aprovechamiento de las herramientas, al interés de los ciudadanos, entre otros (ver por ejemplo Carter y Belanger, 2004; Holzer, Melitski, Rho y Schwester, 2004; Jaeger, 2005; Mossberger y Jimenez, 2009).

Por ello, frente a un exceso de optimismo en las NTICs y un sobredimensionamiento de los potenciales beneficios de las mismas, se pretende un acercamiento renovado que problematice sus posibilidades, al tiempo que haga incidencia en el que creemos es el elemento principal: la ciudadanía. Y es que las nuevas tecnologías de la información y la comunicación son solo herramientas que ofrecen nuevos retos y potencialidades, pero que no obtienen por sí mismas los beneficios que se esperan de su aprovechamiento. Esto es, queda el reto más importante, la 
utilización y el uso adecuado de las mismas (García, 2005; Mossberger y Jimenez 2009; Garcia y Lippez, 2015).

Con esto en mente y considerando los avances de Colombia y su intención de promocionar las NTICs y el gobierno electrónico en todos los niveles de gobierno, es importante sumar miradas al desarrollo de estos esfuerzos gubernamentales. Tales esfuerzos se han venido materializando desde hace varios años, en particular en los dos últimos periodos de gobierno podemos destacar, por un lado, el Plan Vive Digital, formulado en el año 2010 y por otro, el Decreto número 2573 de 2014 que desarrolla los objetivos de la estrategia de Gobierno en Línea, que es la que pone en marcha el gobierno electrónico en el país. Con esto se busca promover un Estado más eficiente a través del aprovechamiento de las Tecnologías de la Información y Comunicación.

La estrategia establece un Manual del Gobierno en Línea que está conformado por cuatro (4) componentes fundamentales que guían la implementación del e-government por parte de entidades del orden nacional y territorial, a saber: TIC para servicios, TIC para el gobierno abierto, TIC para la gestión, seguridad y privacidad de la informaciín. A partir de la definición de estos componentes fundamentales, el gobierno establece unas metas y etapas de implementación de acuerdo a la categoría de las entidades territoriales, se crea el índice de Gobierno Electrónico que mide los avances de la estrategia a nivel nacional y se generan herramientas transversales ${ }^{6}$ para el acompañamiento de las instituciones, entre ellas un mapa de ruta que establece los trámites y servicios que más necesitan los colombianos, y a la vez una asistencia para construir las páginas Web de las entidades. Lo más importante es que se establecen unos plazos dentro de los cuales las entidades nacionales y territoriales están obligadas a cumplir con las metas en un periodo de evaluación que empieza en el año 2015 y se extiende hasta el año 2020.

En este orden de cosas, la estrategia de Gobierno en Línea establece un horizonte de desarrollo con tres objetivos específicos y

6 Las ocho herramientas transversales que deben utilizar las entidades de orden nacional y territorial para la modernización de su gestión son: portal sí virtual, mapa de ruta, carpeta ciudadana, autenticación, datos abiertos, innovación abierta, urna de cristal, y las plataformas web. 
acumulativos: prestar servicios en línea, una mejora en la gestión, un empoderamiento y una mayor confianza de los ciudadanos, todo lo cual debería conducir a construir un Estado más eficiente, más transparente y más participativo (Portal Gobierno en Línea, 2015) ${ }^{7}$. La propia estrategia implementa un índice de gobierno en línea con el objetivo de adelantar un proceso de monitoreo y evaluación que muestra el estado del avance de las entidades en la implementación de la estrategia. Concretamente el índice revisa el avance de la eficiencia electrónica, el gobierno abierto y los servicios, por lo que se sustenta en indicadores que revisan la implementación de las plataformas, la articulación con la gestión de procesos de las entidades y la provisión de información y consulta. Aun cuando el índice incorpora elementos de participación ciudadana, este no es el objetivo del mismo. De allí que la mirada propuesta en este documento que se concentra en la revisión de la orientación al ciudadano para la promoción de la participación activa en condiciones de deliberación consciente, aporta una mirada complementaria a la ya implementada desde la estrategia.

Dados los avances que Colombia ha tenido y las potencialidades otorgadas a las NTICs, nuestra mirada se concentra únicamente en la dimensión de las potencialidades de promoción del compromiso y la participación ciudadana -algunos la llamarian incluso empoderamiento ciudadano- a partir de las herramientas de gobierno electrónico. Nuestro objetivo no es realizar una evaluación de la estrategia a partir de esta dimensión porque como hemos visto, ella se plantea como una meta en el mediano plazo. Por ello nuestra perspectiva entiende el compromiso y el empoderamiento desde el desarrollo de las capacidades deliberativas y de los espacios deliberativos de los asuntos públicos, lo que exige revisar los progresivos avances de la estrategia, pero no solo sobre los indicadores del propio índice, sino a la luz de los exigentes criterios del modelo deliberativo habermasiano. En este contexto, este trabajo revisa los avances realizados a nivel subnacional, en particular a nivel local. Para ello nos propusimos identificar la orientación al ciudadano de los sitios Web de los 32 municipios capitales de departamento en Colombia. A nuestro juicio este análisis

Puede ampliarse esta información en: http:/ / estrategia.gobiernoenlinea.gov.co/623/w3-channel. html 
arroja una visión lo suficientemente rica como para percibir el avance progresivo en la implementación de la estrategia que sirve como una primera aproximación a nivel local en Colombia.

Para nuestro propósito procedemos del siguiente modo: En la siguiente sección abordamos el desarrollo en la concepción del gobierno electrónico y el aprovechamientos de las NTICs en general como herramientas de una posible reconfiguración de las relaciones Estado-sociedad en el siglo XXI, pero particularmente a partir del entendimiento de su papel en la promoción de la deliberación pública, la cual supone un compromiso y una participación ciudadana cualificada. Posteriormente planteamos el diseño metodológico, en el que sobresale la construcción de una batería de indicadores o características a revisar en las estrategias de gobierno electrónico para dar cuenta de la medida en que estas reflejan el aprovechamiento de las NTICs no solo para la provisión de información y la consulta ciudadana, sino fundamentalmente para indagar si estas contribuyen a facilitar y potenciar el compromiso y la participación activa de la ciudadanía. Luego presentamos los principales resultados de nuestro análisis sobre la orientación al ciudadano para las 32 ciudades capitales. Y finalmente, en la última sección resumimos los hallazgos más importantes, los aspectos más significativos del artículo, sus contribuciones más relevantes y sugerimos ideas para futuros trabajos.

\section{Del gobierno electrónico y las relaciones Estado-sociedad en el siglo XXI: las promesas y limitaciones de las NTICs}

Las potencialidades de las NTICs aparecen como una ventana de oportunidad para incidir en las relaciones entre el Estado y la sociedad. Ello es así porque se espera que el aprovechamiento que hagan las organizaciones gubernamentales de estos instrumentos tenga consecuencias importantes en los procesos gubernamentales y fundamentalmente en la interacción de los ciudadanos con dichas organizaciones. Como se verá, al gobierno electrónico en general se le 
endilga la capacidad de impactar positivamente la medida en que los ciudadanos pueden acceder a la información y los servicios gubernamentales, la posibilidad de reducir los costos de las operaciones y los servicios públicos, y de abrir canales de comunicación y participación ciudadana; todo lo cual se sintetiza en mayor transparencia, rendición de cuentas, eficiencia, legitimidad y gobernabilidad, es decir que se constituye en la posibilidad de renovar las relaciones del Estado y la sociedad con mayor colaboración y confianza. Sin embargo, las potencialidades que se otorgan a estas NTICs se perciben sustentadas en las herramientas tecnológicas per se y no tanto en el uso que se haga de estas. Por ello, contrario a una mirada que pueda sobrevalorar el papel de las NTICs, sostenemos que el potencial de estas herramientas depende no solo de disponer de estos canales sino también del uso efectivo que los ciudadanos puedan hacer de estas. Para revisar este asunto con detenimiento se abordan y desarrollan conceptos claves como el gobierno electrónico, el compromiso ciudadano, la democracia digital y la deliberación y participación política.

Con relación a la concepción y el desarrollo del gobierno electrónico, Villoria y Ramírez (2013) identifican al menos cuatro grandes etapas. Una primera etapa se denomina de administración electrónica en sentido estricto, donde se concibe el gobierno electrónico como un instrumento tecnológico que permite mejorar la eficacia y la eficiencia de la actuación del gobierno mediante la entrega de información y la prestación de servicios en línea que favorecen interacciones de los ciudadanos con el Estado más sencillas y cómodas por darse en tiempo real y sin la necesidad de desplazarse físicamente a las oficinas estatales.

En la segunda etapa Villoria y Ramírez (2013) sostienen que el gobierno electrónico se asocia más a una idea de un gobierno transparente y que rinde cuentas. En este caso, el uso de las NTICs va dirigido a proveer información con el objetivo de garantizar mayor transparencia, accountability, imparcialidad e integridad de los gobiernos. En la tercera etapa los autores asocian el gobierno electrónico a una administración inclusiva e imparcial que por tanto, busca reducir las brechas y superar la exclusión. Para ello se resalta la importancia de minimizar las barreras a la accesibilidad, de tal manera que nadie 
quede al margen de sus beneficios. Por último, el uso de las NTICs se asocia a una potencialidad creadora y colaborativa (gobierno electrónico participativo y colaborativo).

De alguna manera esta evolución indica un cambio en la comprensión y el propósito con que los gobiernos pueden usar las NTICs. En consonancia con esta evolución, Mayer-Shonberger y Lazer (2007) 1laman la atención sobre dos maneras distintas de concebir el gobierno electrónico. La primera de ellas, según estos autores, corresponde a una visión estrecha o reducida en tanto entiende el gobierno electrónico en relación con la implementación de las NTICs para la provisión de información, para fomentar las interacciones y las transacciones. Muy similar a los primeros desarrollos o las etapas iniciales identificadas por Villoria y Ramírez (2013). En contraposición, Mayer-Shonberger y Lazer (2007) sugieren una concepción más amplia del gobierno electrónico que hace referencia "al uso entero de las tecnologías de la información y la comunicación en el sector público" (p. 3). De allí que incluso sugieran repensar la denominación de gobierno electrónico o e-government de tal manera que se reemplace por information-government, y así se piense en todos los elementos que suponen la gestión de la información en el sector público. Esto es fundamental considerando que esta perspectiva amplia posibilita pensar en que estas herramientas facilitan el flujo de la información y la comunicación al interior de las organizaciones públicas, entre las organizaciones y de estas con los ciudadanos. Al pensar entonces en flujos de información, se supera una visión reduccionista que solo prevé la disponibilidad de información en sitios web gubernamentales, sin contar con la posibilidad del uso de esa información y de la retroalimentación por parte de los ciudadanos.

Por ello, el gobierno electrónico desde esta perspectiva amplia supone no solo la provisión de información y la facilitación de trámites, sino que además prevé el uso activo de los canales de comunicación por parte de los ciudadanos para incidir en la gestión de lo público. Solo desde este punto de partida podemos comprender mejor la evolución en el uso de las NTICs en relación con la participación ciudadana. La OCDE (2001) argumenta que la participación política debe implicar al menos tres aspectos correlativos: la posibilidad de ser informado, 
los mecanismos para participar en la toma de decisiones y la capacidad de contribuir e influir en la agenda política.

En ese orden de ideas, la OCDE (2001) sugiere tres niveles de participación: (1) la información, que presupone una relación de una sola vía en la que el gobierno produce y suministra información para uso de los ciudadanos; (2) la consulta: una relación bidireccional en la que los ciudadanos proporcionan retroalimentación al gobierno pero en la cual son los gobiernos quienes definen los temas de consulta, establecen las preguntas y gestionan el proceso, mientras que se invita a los ciudadanos a contribuir con sus puntos de vista y opiniones; y (3) la participación activa: una relación basada en la colaboración con el gobierno en la que los ciudadanos participen activamente en la definición del proceso y el contenido de la formulación de políticas. Al alcanzar este tercer nivel de participación, se reconoce en pie de igualdad a los ciudadanos en el establecimiento de la agenda, aunque la responsabilidad de la decisión final recaiga en el gobierno.

Al situarse en este último nivel de participación, o mejor, de participación activa, surgen dos conceptos fundamentales que deben revisarse: el compromiso ciudadano y la democracia digital. El civic engagement o compromiso ciudadano se entiende como el "conocimiento, la discusión, el interés y la participación en asuntos públicos -en el gobierno y la política, en los asuntos de política pública, y en la comunidad" (Mossberger y Jimenez, 2009, p. 2). Este concepto que entendemos como sinónimo del de participación activa mencionado, trasciende connotaciones tradicionales o básicas de participación ciudadana porque supone un involucramiento y una disposición mucho mayor por parte del ciudadano para comprender, analizar y participar de manera consiente y constructiva en los asuntos públicos (OCDE, 2001). Para que este compromiso y participación ciudadano se dé es preciso que exista antes una conciencia y un conocimiento de los ciudadanos sobre lo que hace el gobierno, sobre qué hacen las diferentes organizaciones públicas, sus responsabilidades, etc., por lo que la información que se provea para educar a los ciudadanos al respecto se constituye en una condición básica para la participación o el compromiso ciudadano. 
Hablar de compromiso ciudadano remite directamente al concepto de e-democracy y en particular, a la idea de deliberación. Deliberar es dar y pedir razones, a favor o en contra de nuestras acciones y creencias. La democracia deliberativa aspira por lo tanto a adoptar las decisiones políticas mediante un proceso intersubjetivo de carácter argumentativo en condiciones de razonamiento libre y público entre iguales por aquellos que son gobernados por las decisiones (Cohen, 2001; Habermass, 2005). En el modelo democrático habermasiano, el aspecto clave no es tanto la participación sino la deliberación, entendida ahora ya como una participación cualificada donde la democratización y la resolución de los problemas de nuestras democracias pasa necesariamente por una ciudadanía activa pero también necesariamente, por la creación de espacios y esferas de debate público. Esto implica que la deliberación en el sentido habermassiano no solo incluye la participación per se, sino la exigencia de que cada participante deberá tener la misma posibilidad de ser escuchado, introducir temas, propuestas y enfoques, y cuyas opiniones sean públicas y estén libres de cualquier coerción externa o interna (Habermas, 2005). Solo así tiene sentido plantearnos en qué medida la tecnología, sus diferentes componentes y sus múltiples utilidades, podrian ayudar a hacer más interactivo y eficiente este proceso.

Particularmente los gobiernos locales pueden usar sus sitios Web para educar a los ciudadanos e involucrarlos en los asuntos públicos a través de la provisión de información. De acuerdo con Holzer et al. (2004), la provisión de la información puede hacerse de manera estática o dinámica. En el primer caso la comunicación se da en una sola vía, por lo que los sitios Web se limitan a la provisión de información, sin dar oportunidad para la reacción de los ciudadanos, lo que sí ocurre cuando la información se provee de manera dinámica, cuando se promueve una comunicación en doble vía.

Con la información suficiente para conocer cómo funciona el gobierno, es posible entonces que los ciudadanos participen en la deliberación y toma de decisiones públicas, de allí que surja entonces el concepto mencionado de la democracia digital o e-democracy. Este concepto incluiría "todas las prácticas para fomentar valores democráticos 
usando las tecnologías de la información y la comunicación" (Holzer et al., 2004, p. 8). Como ocurre con la provisión de información, en términos de participación Holzer et al. (2004) asegura, que en el proceso de toma de decisiones la participación también puede promoverse de manera estática, que se da cuando hay algún tipo de consulta a los ciudadanos pero no un productivo debate o deliberación, o de manera dinámica cuando se promueve dicha deliberación al estilo habermasiano donde ciudadanos, grupos de interés, los medios de comunicación, los partidos políticos y demás, debaten sobre asuntos públicos y se da una "amplia y libre interacción entre los participantes" (Holzer et al., 2004, pp. 13-14). Así podemos comprender entonces el concepto de e-deliberación, como aquel que ocurre cuando se promueve la participación dinámica, es decir la deliberación ciudadana -emanada de su compromiso y facilitada por la información provista- a través de las NTICs, particularmente a través de medios electrónicos.

Para promover dicho contexto de fructífero compromiso ciudadano y de deliberación pública, es importante señalar los avances o las oportunidades que ofrece la denominada Web 2.0 o red interactiva, ya que sus herramientas "permiten: 1) personalizar y compartir información a través de $[. .$.$] alertas de correo electrónico y redes sociales [por$ ejemplo]; y 2) la creación de contenidos por medio de blogs [...] y wikis [por ejemplo]" (Mossberger y Jimenez, 2009, p. 9). De allí que sea posible identificar o asociar diferentes herramientas o tecnologias a determinados niveles de participación, en otras palabras, las herramientas facilitarian un objetivo de participación pretendido (ODCE, 2001; Al Dalou y Shanab, 2013).

Sin embargo, la oportunidad de generar un compromiso y una deliberación ciudadana a partir del aprovechamiento de las NTICs no debe conducir a un exagerado optimismo. Como Mossberger y Jimenez (2009) advierten, "los sitios web gubernamentales pueden facilitar pero no crean [no producen] el compromiso ciudadano" (p. 6), y Holzer et al. resaltan que "no hay evidencia que sugiera que la calidad de la toma de decisiones se mejore o que las decisiones sean más democráticas dada la integración de NTICs y aplicación digitales" (p. 10). Además, la literatura sobre la brecha digital que aparece como una 
restricción estructural en el acceso, la accesibilidad y la usabilidad de los sitios Web, es profusa (ver por ejemplo Hacker, Mason y Morgan, 2009; Helbig, Gil-García y Ferro, 2009; Rubaii-Barrett y Wise, 2006; 2008). Y otros incluso han llamado la atención sobre la disposición ciudadana a usar las herramientas como un elemento que debe ser considerado para garantizar un adecuado aprovechamiento de las NTICs (Carter y Belanger, 2004). Debemos recordar entonces que son las personas quienes usan o no las tecnologías, son quienes deliberan sobre los asuntos públicos, quienes toman decisiones y quienes tienen el compromiso ciudadano y materializan la democracia digital a partir del uso adecuado de las herramientas.

\section{Diseño metodológico}

En la perspectiva de contribuir a las miradas que revisan el desarrollo y los avances de la implementación del gobierno electrónico en Colombia a nivel local y desde la perspectiva de identificar la medida en que este desarrollo favorece el compromiso y la deliberación ciudadana, nos hemos propuesto adelantar la revisión de los sitios Web de las alcaldías y concejos de los municipios capitales de los 32 departamentos de la República de Colombia. La selección de los 32 municipios considera que en su carácter de capitales de los departamentos, dichos municipios pueden llevar la batuta o ser pioneros en la implementación de la estrategia de Gobierno en Línea en sus respectivos departamentos.

En la revisión de los sitios Web municipales realizada durante el mes de junio de 2015, se buscó identificar la presencia de 47 características (herramientas web, aplicaciones, información, o documentos, cuya lista se encuentra en la Tabla No. 1) que sintetizan lo sugerido en la literatura (ver por ejemplo Al-Dalou y Abu-Shanab, 2013; Criado y Ramio,, 2003; Mossberger y Jimenez ,2009; OCDE, 2001, United Nations, 2014) como herramientas que podrian facilitar las tres dimensiones señaladas por la OCDE para la promoción de la participación ciudadana, es decir, la provisión de información, la consulta y la participación activa o el compromiso ciudadano. En cuanto a la provisión 
de información, se evaluó la presencia de 31 items, entre los que se destacan la publicación de información de contacto de las autoridades y los servidores públicos locales, la publicación de documentos de planificación del desarrollo, el territorio y las finanzas locales, así como de versiones 'ciudadanas' de esos documentos que se caracterizan por ser sucintas y comprensibles para los ciudadanos del común, y la presencia de 16 ítems asociados específicamente a ejercicios de rendición electrónica de cuentas.

En cuanto a la segunda dimensión, es decir el aprovechamiento de herramientas electrónicas que faciliten la consulta a los ciudadanos, se revisaron ocho aspectos, entre los que se destacan las herramientas que facilitan la comunicación en redes sociales y la disposición de encuestas virtuales, entre otros. Finalmente, en cuanto a la tercera dimensión relacionada con la participación activa o de compromiso ciudadano, se contabilizó el uso de ocho herramientas web para la innovación y la co-creación, para la retroalimentación, evaluación y la deliberación en torno a políticas y programas públicos y más aún, se revisó la medida en que las herramientas están siendo efectivamente usadas. Todo ello haciendo especial hincapié en la medida en que su diseño real provea de los recursos e instrumentos a la ciudadanía para fomentar la 'e-deliberación'.

Se parte entonces de una revisión que dé cuenta de la medida en que las 47 herramientas para la participación ciudadana están siendo implementadas en los sitios web de los 32 municipios capitales de departamento en Colombia, por ello lo que se busca es identificar los dispositivos que más o menos se usan, de tal manera que se puedan señalar caminos que permitan fortalecer la estrategia de gobierno electrónico en la perspectiva de facilitar la e-deliberación. Luego se revisa de manera agregada la medida en que los 32 municipios están implementando herramientas para la provisión de información, la consulta y el compromiso o la participación ciudadana. Por el momento en que se realiza el estudio y lo planteado en la estrategia de Gobierno en Linea descrita anteriormente, se espera que por ahora se hayan implementado más herramientas de provisión de información que de consulta y deliberación. Finalmente, el ejercicio se concentra 
en verificar la medida en que cada uno de esos 32 municipios capitales de departamento han adoptado las 47 herramientas revisadas, generando insumos que permitan tener una idea sobre el estado de implementación de la estrategia en dichos municipios, así como resaltando los territorios en lo que hay mayor o menor avance en la misma.

Ciertamente no se tiene una pretensión de generalización con base en los hallazgos, no buscamos establecer el estado del desarrollo de la implementación de la estrategia en todos los municipios del país y sus alcances en materia de la promoción y la participación ciudadana a partir de esta primera aproximación; aunque debe reconocerse que en todo caso los municipios capitales son los de mayores capacidades en el contexto de cada uno de sus departamentos, por lo que su revisión puede ofrecer una interesante mirada sobre el aprovechamiento que los gobiernos municipales hacen de estas herramientas.

Debe advertirse además que el estudio tampoco pretende establecer relaciones de causalidad que indiquen las variables que explican el nivel de implementación de la estrategia o la medida en que los ciudadanos hagan uso de las herramientas de gobierno electrónico en sus territorios. Dado que se trata de una primera aproximación, el estudio sí podría orientar posteriores ejercicios que, teniendo en cuenta los resultados de este como insumo, sugiera explicaciones sobre las causas del uso y aprovechamiento de las herramientas. De alli que sea preciso enfatizar que el carácter del estudio propuesto es solamente exploratorio, ya que lo que se busca es simplemente dar cuenta de la medida en que los 32 municipios capitales de departamento en Colombia han venido implementando herramientas web que faciliten la provisión de información, la consulta, el compromiso y la participación ciudadana. Con ello, sería posible tener una sensación de la medida en que se avanza hacia un nuevo estado de cosas en términos de la relación Estado-ciudadanía, si efectivamente estamos andando el camino de reconfigurar la relación con el Estado a partir de las promesas del gobierno electrónico y en general de las NTICs. 


\section{Presentación de resultados: Orientación al ciudadano de los sitios web de los municipios capitales de departamento en Colombia}

A continuación se presenta la Tabla No. 1 que sintetiza la presencia de cada uno de los 47 rasgos señalados para identificar la provisión de información, la consulta y el compromiso y la participación activa ciudadana en el conjunto de los 32 sitios Web revisados.

\begin{tabular}{|c|c|c|c|}
\hline $\begin{array}{l}\text { Orientación } \\
\text { al ciudadano }\end{array}$ & Indicadores & n..$^{\circ}$ & $\%$ \\
\hline \multirow{25}{*}{$\begin{array}{l}\text { Provisión de } \\
\text { información }\end{array}$} & Información de contacto Administración & 29 & $91 \%$ \\
\hline & Información de contacto Concejales & 12 & $38 \%$ \\
\hline & Opción de traducción de la página & 24 & $75 \%$ \\
\hline & Herramientas para personas discapacitadas & 2 & $6 \%$ \\
\hline & Plan de Desarrollo disponible & 30 & $94 \%$ \\
\hline & $\begin{array}{l}\text { Hay PDM 'ciudadano' (versión sucinta para revisión rápida y } \\
\text { comprensible del ciudadano común) }\end{array}$ & 11 & $34 \%$ \\
\hline & Se encuentra POT o EOT & 24 & $75 \%$ \\
\hline & $\begin{array}{l}\text { POT 'ciudadano' (versión sucinta para revisión rápida y } \\
\text { comprensible del ciudadano común). }\end{array}$ & 4 & $13 \%$ \\
\hline & Se encuentra MFMP & 9 & $28 \%$ \\
\hline & $\begin{array}{l}\text { Hay Marco Fiscal de mediano plazo ciudadano (versión sucinta para } \\
\text { revisión rápida y comprensible del ciudadano común) }\end{array}$ & 0 & $0 \%$ \\
\hline & Publicación de decretos & 28 & $88 \%$ \\
\hline & Publicación de acuerdos & 24 & $75 \%$ \\
\hline & Se encuentra Plan Operativo Anual de Inversiones & 13 & $41 \%$ \\
\hline & Se encuentran presupuestos 2012-2015 & 14 & $44 \%$ \\
\hline & $\begin{array}{l}\text { Hay presupuestos 'ciudadanos' (versión sucinta para revisión rápida } \\
\text { y comprensible del ciudadano común) }\end{array}$ & 0 & $0 \%$ \\
\hline & Hay informe, presentación, video o documento de Rendición de Cuentas & 26 & $81 \%$ \\
\hline & Es fácil de encontrar (a un click del portal principal) & 22 & $69 \%$ \\
\hline & Posee información integrada de todos los sectores de gobierno & 19 & $59 \%$ \\
\hline & La información es comprensible para el ciudadano común & 19 & $59 \%$ \\
\hline & Usa estadisticas & 17 & $53 \%$ \\
\hline & $\begin{array}{l}\text { La información se presenta con relativa frecuencia para facilitar } \\
\text { comparabilidad }\end{array}$ & 14 & $44 \%$ \\
\hline & $\begin{array}{l}\text { Se usan categorías estandarizadas en cada período para facilitar } \\
\text { comparación }\end{array}$ & 14 & $44 \%$ \\
\hline & Hay un archivo de reportes de Rendición de Cuentas & 23 & $72 \%$ \\
\hline & $\begin{array}{l}\text { La extensión de los documentos facilita y motiva su revisión por } \\
\text { parte del ciudadano del común }\end{array}$ & 18 & $56 \%$ \\
\hline & $\begin{array}{l}\text { Hay una función que permite la búsqueda al interior de los } \\
\text { documentos }\end{array}$ & 13 & $41 \%$ \\
\hline
\end{tabular}




\begin{tabular}{|c|c|c|c|}
\hline \multirow{6}{*}{$\begin{array}{l}\text { Provisión de } \\
\text { información }\end{array}$} & $\begin{array}{l}\text { Hay funciones o herramientas que permiten compartir los } \\
\text { documentos }\end{array}$ & 4 & $13 \%$ \\
\hline & $\begin{array}{l}\text { Hay funciones o herramientas que permiten a los ciudadanos opinar } \\
\text { sobre los documentos }\end{array}$ & 1 & $3 \%$ \\
\hline & $\begin{array}{l}\text { Hay funciones o herramientas que permiten a los ciudadanos } \\
\text { 'deliberar' sobre los documentos de Rendición de Cuentas }\end{array}$ & 0 & $0 \%$ \\
\hline & $\begin{array}{l}\text { Hay funciones o herramientas que notifican a los participantes } \\
\text { sobre una nueva participación sobre los documentos de Rendición } \\
\text { de Cuentas }\end{array}$ & 4 & $13 \%$ \\
\hline & $\begin{array}{l}\text { Hay foros o salas de discusión sobre los documentos de Rendición } \\
\text { de Cuentas }\end{array}$ & 2 & $6 \%$ \\
\hline & Hay encuestas sobre los documentos de Rendición de Cuentas & 5 & $16 \%$ \\
\hline \multirow{8}{*}{ Consulta } & Comunicación en redes sociales & 26 & $81 \%$ \\
\hline & Espacios de discusión & 15 & $47 \%$ \\
\hline & Hay motor de búsqueda & 28 & $88 \%$ \\
\hline & Buscador de documentos archivados & 11 & $34 \%$ \\
\hline & Encuestas online & 19 & $59 \%$ \\
\hline & Evaluaciones & 3 & $9 \%$ \\
\hline & Enlaces a participación de poblaciones & 3 & $9 \%$ \\
\hline & Mapas o documentos para consulta territorio & 24 & $75 \%$ \\
\hline \multirow{8}{*}{$\begin{array}{l}\text { Participación } \\
\text { activa o de } \\
\text { compromiso } \\
\text { ciudadano }\end{array}$} & Plataformas de innovación y co-creación & 3 & $9 \%$ \\
\hline & Se usa o no en período de gobierno & 3 & $9 \%$ \\
\hline & Evaluación de políticas, programas y gestión & 14 & $44 \%$ \\
\hline & Se usa o no en período de gobierno & 13 & $41 \%$ \\
\hline & Herramientas de comunicación & 18 & $56 \%$ \\
\hline & Se usa o no en período de gobierno & 14 & $44 \%$ \\
\hline & Eventos off line (calendarios, comunicados de prensa, etc.) & 26 & $81 \%$ \\
\hline & Fechas y horas de las sesiones de concejos & 6 & $19 \%$ \\
\hline
\end{tabular}

Tabla 1. Uso de herramientas Web para la promoción de la participación ciudadana en los 32 municipios capitales de departamento en Colombia

Fuente: Elaboración propia

A partir de los resultados de la Tabla No. 1 se puede establecer que en promedio los rasgos o las características revisadas aparecen en el $43 \%$ de los casos estudiados. Se destacan que de los 32 sitios Web, en 94\% el Plan de Desarrollo del Municipio es puesto a disposición de los ciudadanos y en $91 \%$ hay información de contacto con la administración. Paralelamente aquellos ítems en los que hay un avance mucho más discreto son por ejemplo, la presencia de herramientas para personas con discapacidades que solo figura en $6 \%$ de los casos, la disposición de los Planes de Ordenamiento Territorial ciudadanos -es decir resumidos, 
sistematizados y provistos de manera comprensible para los ciudadanos del común- que apenas se difunden en 13\% de los sitios Web, o los Marcos Fiscales de Mediano Plazo para ciudadanos y presupuestos ciudadanos que no se encuentran en ninguna de las páginas revisadas. También se extraña el uso de herramientas que permitan la reacción de los ciudadanos frente a los documentos y ejercicios de rendición de cuentas, tales como chats, foros, encuestas y otros (ver Tabla No. 1). Finalmente también se resalta la poca presencia de enlaces que orienten a los ciudadanos hacia grupos de interés por minorias o sectores, o información relacionada con participación de determinados grupos que solo se observa en 9\% de los casos, lo cual ocurre con el aprovechamiento de plataformas destinadas a la co-creación de políticas públicas.

Por su parte, la Tabla No. 2 que se presenta a continuación refleja el desempeño municipal agregado en las tres categorias identificadas orientadas a la participación ciudadana. Los resultados de cada categoría corresponden al porcentaje de herramientas o características correspondientes a cada grupo o categoría que están presentes en los sitios Web revisados, respecto al uso potencial de todas las características en todos los casos.

\begin{tabular}{|l|c|}
\hline \multicolumn{1}{|c|}{ Categorias o niveles para la participación } & $\%$ \\
\hline Provisión de información & $43 \%$ \\
Informes de Rendición de cuentas & $39 \%$ \\
\hline Consulta & $50 \%$ \\
\hline Participación activa o compromiso ciudadano & $38 \%$ \\
\hline
\end{tabular}

Tabla 2. Disposición de herramientas para la provisión de información, la consulta y la participación ciudadana en los 32 sitios Web de los municipios capitales de departamento en Colombia

Fuente: elaboración propia

Como era de esperarse, en el diseño de las páginas oficiales de los municipios estudiados se observa una mayor disposición de elementos que permiten la provisión de información que de aquellos que facilitan la participación activa de los ciudadanos. De estos resultados resumidos en la Tabla No. 2 además se destaca que, aunque efectivamente existe la intencionalidad de implementar diferentes mecanismos de consulta a 
los ciudadanos, los canales dispuestos para que los ciudadanos expresen sus opiniones resultan limitados a esa dimensión de consulta y no tanto al desarrollo más extendido de una participación activa plena. Por ejemplo, debe mencionarse que en la búsqueda realizada, aunque efectivamente los municipios adoptan $50 \%$ de las herramientas que podrian implementar para la consulta ciudadana, también fue persistente encontrar que las mismas podían no utilizarse, eran utilizadas muy ocasionalmente o se usaban para conocer percepciones relacionadas con asuntos menores o poco trascendentes en las discusiones de política pública.

Desafortunadamente el instrumento desarrollado para adelantar este trabajo no permitía recoger información que valorara la calidad del debate público ni tampoco el uso de las herramientas en términos cuantitativos, apenas su disposición en las páginas web. En todo caso estos indicios sí señalan la necesidad de avanzar en nuevos ejercicios que vuelquen su mirada, más allá de la orientación al ciudadano, en el uso que tanto gobierno como ciudadanos hacen de las herramientas de gobierno electrónico.

Al revisar los resultados de las tablas 1 y 2 en conjunto, también resulta interesante mencionar que aunque hay un esfuerzo por proveer información a los ciudadanos, no resulta claro que esta información se entregue en condiciones que permitan una sencilla comprensión, ni que por tanto faciliten una subsecuente participación ciudadana. Ejemplo de ello es prácticamente la inexistencia de instrumentos de planificación 'ciudadanos' que entreguen versiones sintetizadas de los mismos, la escasa adopción de rasgos de un adecuado ejercicio de rendición de cuentas y el muy bajo uso de herramientas que permitan la reacción y el debate a partir de dichos instrumentos de rendición de cuentas. Es probable que esto se explique en la práctica generalizada de rendir cuentas a través de audiencias públicas presenciales y no tanto por medios electrónicos, que parecen utilizarse apenas para convocar a las mencionadas audiencias o para publicar información sobre la que, en todo caso, no se tienen espacios para deliberar. Estos elementos también llaman la atención sobre los cambios que se requieren en términos de prácticas, rutinas y cultura -tanto desde lo organizacional como de lo político- para hacer un aprovechamiento pleno de las oportunidades que estas herramientas ofrecen. 
Por municipios, a continuación se presenta la Tabla No. 3 que sintetiza la adopción de herramientas de gobierno electrónico que facilitarian la participación ciudadana en los sitios web de los municipios revisados:

\begin{tabular}{|c|c|c|}
\hline Ciudad & Caracteristicas presentes & $\%$ (de 47 posibles) \\
\hline Tunja & 32 & $68 \%$ \\
\hline Medellin & 32 & $68 \%$ \\
\hline Bogotá & 29 & $62 \%$ \\
\hline Florencia & 28 & $60 \%$ \\
\hline Bucaramanga & 28 & $60 \%$ \\
\hline Yopal & 28 & $60 \%$ \\
\hline Pasto & 26 & $55 \%$ \\
\hline Cali & 25 & $53 \%$ \\
\hline Manizales & 24 & $51 \%$ \\
\hline Barranquilla & 23 & $49 \%$ \\
\hline Riohacha & 22 & $47 \%$ \\
\hline Neiva & 22 & $47 \%$ \\
\hline Quibdó & 22 & $47 \%$ \\
\hline San José del Guaviare & 20 & $43 \%$ \\
\hline Leticia & 20 & $43 \%$ \\
\hline Mocoa & 20 & $43 \%$ \\
\hline Cúcuta & 19 & $40 \%$ \\
\hline Pereira & 19 & $40 \%$ \\
\hline Puerto Inirida & 19 & $40 \%$ \\
\hline Valledupar & 18 & $38 \%$ \\
\hline Puerto Carreño & 18 & $38 \%$ \\
\hline Sincelejo & 17 & $36 \%$ \\
\hline Popayán & 16 & $34 \%$ \\
\hline Armenia & 16 & $34 \%$ \\
\hline Villavicencio & 16 & $34 \%$ \\
\hline Ibagué & 16 & $34 \%$ \\
\hline Arauca & 15 & $32 \%$ \\
\hline Mitú & 15 & $32 \%$ \\
\hline Cartagena & 14 & $30 \%$ \\
\hline San Andrés & 12 & $26 \%$ \\
\hline Monteria & 10 & $21 \%$ \\
\hline Santa Marta & 10 & $21 \%$ \\
\hline
\end{tabular}

Tabla 3. Adopción de herramientas Web para la promoción de la participación ciudadana por municipios capitales en Colombia

Fuente: elaboración propia 
Los resultados consignados en la tabla anterior dan luces a los municipios sobre el estado de avance que cada uno tiene en el camino de aprovechar las herramientas de gobierno electrónico para la participación ciudadana. No se trata de establecer una suerte de ranking o de señalar ganadores o perdedores, pero con la información presentada las autoridades del gobierno central sí podrian identificar las entidades territoriales que requieren mayor apoyo en la implementación de la estrategia, y posteriores ejercicios podrian orientarse a la revisión de estudios de caso que destaquen experiencias exitosas en los municipios que tengan una mayor implementación de herramientas, o que identifiquen obstáculos en aquellos municipios que tengan un tímido avance en la adopción de las mismas.

\section{Reflexiones finales}

Como mencionábamos, desde una concepción no reduccionista del gobierno electrónico, el uso de las NTICs no solo viene limitado a la provisión de información y la facilitación de trámites, sino que además se prevé el uso activo de los canales de comunicación por parte de los ciudadanos para incidir en la gestión de lo público. Desde este punto de partida la OCDE (2001) identificó tres aspectos correlativos a la participación ciudadana que las NTICs tienen la potencialidad de promover, estos son: la posibilidad de ser informado o de proveer información a los ciudadanos, la posibilidad de estos de retroalimentar el proceso a través de la consulta sobre sus percepciones, o bien el establecimiento de los mecanismos para participar en la toma de decisiones y contribuir e influir en la formación de la agenda política (participación activa o compromiso ciudadano). En este orden de cosas se diseñó una batería de 47 indicadores clasificados sobre estas tres dimensiones mencionadas y se revisó la medida en que estas herramientas están siendo utilizadas en los sitios Web de los 32 municipios capitales de departamento en Colombia. Todo ello haciendo especial hincapié en la medida en que su diseño real provea de los recursos e instrumentos a la ciudadanía para fomentar la 'e-deliberación' y que por esta vía, se esté avanzando hacia una posible reconfiguración de las relaciones ciudadanas 
con el Estado, las cuales se sustentarian en las posibilidades ofrecidas por las NTICs en el gobierno o gobierno electrónico.

El análisis arrojó algunos resultados importantes. Como era previsible dado el grado de avance de la estrategia de Gobierno en Línea en Colombia, era de esperar que los mayores avances se registrasen en los aspectos directamente relacionados con la provisión de información y con la consulta, pero mucho menos en el tema de la promoción de la participación activa. Los resultados confirmaron esta hipótesis. Sin embargo, cabe resaltar algunos hallazgos particularmente importantes.

En primer lugar, aun cuando es cierto que en términos de provisión de información se registran los mayores avances, se observó que son muy escasos los municipios que entregan información en un formato asequible y comprensible para el ciudadano común, por lo que es preciso incrementar esfuerzos para mejorar la provisión de información tanto en cantidad como en calidad. No será posible una verdadera deliberación ciudadana si la información a la que se tiene acceso no es suficiente o no permite ser comprendida por quienes la consulten, de allí que sea fundamental avanzar en garantizar estos elementos que condicionan una participación ciudadana cualificada.

En segundo lugar, aun cuando existen avances importantes en términos de posibilitar la consulta a los ciudadanos, los análisis muestran un muy discreto avance en términos de rendición de cuentas. Es así porque en general se observó que aunque los municipios suelen proveer la información específica sobre lo realizado en audiencias de rendición de cuentas, hay una presencia muy escaza de herramientas que permitan un adecuado ejercicio de retroalimentación por parte de la ciudadanía respecto a tales ejercicios. Además, es muy importante que la consulta, aunque orientada por las administraciones locales, debe permitir el debate cualificado de los ciudadanos sobre las opciones y las decisiones de política pública y no solo deben circunscribirse a la disposición de encuestas insulsas. 
Por último y como era de esperarse, los avances registrados en el establecimiento de herramientas de participación activa son muy tímidos y cuando hay herramientas dispuestas para ello, su uso resulta muy escaso. Se destacan excepciones como la experiencia de Medellín en materia de promoción de la discusión o deliberación sobre política pública, o de co-creacion. En general entonces, aunque hay avances importantes en materia de provisión de información por medios electrónicos, los sitios Web de los 32 municipios capitales de departamento en Colombia aún deben desarrollarse más para generar espacios de debate y deliberación en torno a políticas y decisiones públicas, y más aún debe promover el uso efectivo de tales herramientas, de manera que efectivamente se impacte de manera positiva las relaciones entre el Estado y la ciudadanía a través de una mayor transparencia, eficiencia y rendición de cuentas estatales, y de una mayor incidencia ciudadana en las políticas y las decisiones públicas.

Desde un marco deliberativo de la democracia, la participación en línea de los ciudadanos ha de ser juzgada no en términos cuantitativos, sino sobre todo cualitativos. Esto es, los sitios Web pueden ser canales que sirvan para fomentar la participación en el proceso de toma de decisiones, pero desde una perspectiva deliberativa, esto no solo se garantiza ofreciendo información y canales, sino que depende en última instancia del uso adecuado de estas herramientas por parte de los ciudadanos. Así las cosas, la expectativa de un cambio drástico en las relaciones Estado-sociedad a partir del aprovechamiento de las Nuevas Tecnologías de la Información y la Comunicación para la promoción de una ciudadanía activa en la deliberación pública, parece tener aún un camino largo por recorrer antes de materializarse.

\section{Referencias}

Al-Dalou, R. y Abu-Shanab, E. (2013). E-participation levels and technologies. 6th International Conference on Information Technology (ICIT 2013). Ponencia presentada en Amman, Jordan, 8-10 de mayo (pp. 1-8). Recuperado de http:// sce.zuj.edu.jo/icit13/images /Camera\%20Ready/E-Technology/656.pdf 
Carter, L. y Belanger, F. (2004, enero). Citizen adoption of electronic government initiatives. In System Sciences, 2004. Proceedings of the 37th Annual Hawaii International Conference on IEEE (p. 10). Hawaii. University of Hawai'i at Manoa

Cohen, J. (2001). Democracia y Libertad. En Elster, J. (Ed.), La democracia deliberativa (pp. 235-287). Barcelona: Gedisa.

CRC (2014). Avance de Colombia en la Sociedad de la Información. Informe Sociedad de la Información. Recuperado de http://colombiatic.mintic.gov. co/602/articles-6807_archivo_pdf.pdf

Criado, J. I. y Ramio, C. M. (2003). E-government in practice: an analysis of web site orientation to the citizens in Spanish municipalities. International Journal of Public Sector Management, 16(3), 191-218.

García, G. E. (2005). La democracia electrónica: un análisis desde la teoría política. Documento presentado en el VII Congreso Español de Ciencia Política y de la Administración llevado a cabo en Madrid, España, 21, 22 y 23 de septiembre de 2005.

Garcia, R., y Líppez-De Castro, S. (2015). Technology helps, people make: A smart city governance framework grounded in deliberative democracy. En GilGarcia, R., Pardo, T. A. y Nam, T. (Eds.). Innovation as the New Urban Agenda: A Comprehensive view of the 21st Century Smart City (pp.333-347). USA: Springer.

Hacker, K. L., Mason, S. M. y Morgan, E. L. (2009). Digital disempowerment in a network society. International Journal of Electronic Government Research, 5(2), 57-71. Recuperado de http://search.proquest.com/ docview $/ 222699052$ ?accountid $=13250$

Helbig, N., Gil-Garcia, J. R. y Ferro, E. (2009). Understanding the complexity of electronic government: Implications from the digital divide literature. Government information Quarterly, 26, 89-97.

Holzer, M., Melitski, J., Rho, S. y Schwester, R. (2004). Restoring trust in government: the potential of digital citizen participation. USA: E-Government Series, IBM Center for The Business of Government. 
Habermas, J. (2005). Concluding Comments on Empirical Approaches to Deliberative Politics, Acta Política, 40, 384-392.

Jaeger, P. T. (2005). Deliberative democracy and the conceptual foundations of electronic government. Government Information Quarterly, 22, 702-719.

Kang, S. y Norton, H. E. (2004). Nonprofit organizations' use of the World Wide Web: are they sufficiently fulfilling organizational goals? Public Relation Review, 30, 279-284.

Mayer-Schonberger, V. y Lazer, D. (2007). From electronic government to information government. En Mayer-Schonberger y Lazer (Eds.), Governance and Information Technology (pp. 1- 9). Cambridge: The MIT Press.

Mossberger, K. y Jimenez, B. (2009). Can e-government promote civic engagement? A study of local government websites in Illinois and the U.S. Institute for Policy and Civic Engagement. Chicago: University of Illinois.

Lee, M. (2004). E-reporting: Strengthening democratic accountability. USA: IBM Center for The Business of Government.

OCDE (2001). Citizens as Partners. Information, consultation, and public participation in policy making. Paris: OECD. Recuperado de http: / /www.oecd-ilibrary. org/docserver/download /4201131e.pdf?expires=1435076834\&id=id\&accname=id15861\&checksum=2B2313630BEA54DF1FD511B4E55792A3.

Rubaii-Barrett, N. y Wise, L. R. (2006). Language minorities and the digital divide: A study of state E-government accessibility. Journal of Public Management \& Social Policy, 5-27.

Rubaii-Barrett, N. y Wise, L. R. (2008). Disability access and e-government an empirical analysis of state practices. Journal of Disability Policy Studies, 19(1), 52-64.

United Nations, Department of Economic and Social Affairs (2014). United Nations E-government Survey 2014: E-government for the future we want. Recuperado de http://unpan3.un.org/egovkb/Portals/egovkb/Documents / un/2014-Survey/E-Gov_Complete_Survey-2014.pdf 
Villoria, M. y Ramírez, A. (2013). Los modelos de gobierno electrónico y sus fases de desarrollo. Un análisis desde la Teoría política Gestión y politica pública, 9-103.

\section{Cómo citar este artículo}

Líppez-De Castro, S. y García, R. (2016). Ciudadanos y gobierno electrónico: la orientación al ciudadano de los sitios Web municipales en Colombia para la promoción de la participación. Universitas Humanística, 82, 279-304. http:// dx.doi.org/10.11144/Javeriana.uh82.cgeo 